\title{
Effectiveness of TC-325 (Hemospray) for treatment of diffuse or refractory upper gastrointestinal bleeding - a single center experience
}

\section{다(1)이우}

\author{
Authors \\ Oscar Cahyadi ${ }^{1}$, Markus Bauder ${ }^{1}$, Benjamin Meier ${ }^{1}$, Karel Caca ${ }^{1}$, Arthur Schmidt $^{1,2}$
}

Institutions

1 Department of Gastroenterology and Oncology, Klinikum Ludwigsburg, Ludwigsburg, Germany

2 Department of Medicine II, Medical Center - University of Freiburg, Faculty of Medicine, University of Freiburg, Germany

submitted 14.5.2017

accepted after revision 24.7.2017

Bibliography

DOI https://doi.org/10.1055/s-0043-118794 |

Endoscopy International Open 2017; 05: E1159-E1164

(c) Georg Thieme Verlag KG Stuttgart · New York

ISSN 2364-3722

Corresponding author

Arthur Schmidt, MD, Department of Medicine II, Medical

Center - University of Freiburg, Faculty of Medicine

University of Freiburg, Hugstetter Straße 55, D-79106

Freiburg, Germany

Fax: +49 761 270-25422

arthur.schmidt@uniklinik-freiburg.de

\section{ABSTRACT}

Background and study aims TC-325 (Hemospray, Cook Medical) is a powder agent for endoscopic hemostasis in patients with upper gastrointestinal bleeding (UGIB). Although most publications are based on case-reports and retrospective studies, data on efficacy are promising. Here we report our experience with TC-325 for diffuse or refractory UGIB.
Patients and methods Data on patients receiving TC-325 for endoscopic hemostasis from November 2013 to February 2017 at our center were analyzed retrospectively. Primary endpoints were technical success (successful immediate hemostasis) and clinical success (effective hemostasis and no recurrent bleeding). Secondary endpoints were recurrent bleeding within 3 and 7 days, hospital mortality and TC-325 associated complications. TC-325 was used for bleeding not amenable to standard endoscopic treatment (e.g. diffuse bleeding) or as salvage therapy after failure of conventional methods

Results Fifty-two patients received TC-325 treatment. Most of the patients were treated for peptic ulcer bleeding (18/52 patients, $34.6 \%$ ) and post-interventional bleeding (13/52 patients, $25 \%$ ). Hemospray was used in $23 / 52$ (44.2\%) patients as monotherapy and in 29/52 (55.8\%) patients as a salvage therapy. Application of the powder on the bleeding source was successful in all patients with no therapy-related adverse events (AEs). Immediate hemostasis was achieved in 51/52 (98.1\%) patients. Recurrent bleeding within 3 and 7 days was observed in 22/51 and $25 / 51$ patients respectively ( $43.1 \%$ and $49 \%$ ). The overall clinical success was $56.9 \%$ on day 3 and $51 \%$ on day 7 . Total mortality was $15.4 \%$ (8 patients), bleeding associated mortality was $3.8 \%$ (2 patients). There were no therapy-related AEs.

Conclusions TC-325 showed a high technical success rate as monotherapy for bleeding sources not amenable to standard methods or as an "add-on" therapy after unsuccessful hemostasis. However, rebleeding was frequent in this cohort and further studies are warranted to exactly define a treatment algorithm for TC-325 use.

\section{Introduction}

Upper gastrointestinal bleeding (UGIB) is a common cause for hospitalization with an worldwide annual incidence of about 40 to 150 per 100.000 and significant morbidity and mortality [1]. The most frequent causes of UGIB are peptic ulcers (28$59 \%$ ), mucosal erosive disease of the esophagus or stomach/ duodenum (1-47\%), Mallory-Weiss-syndrome (4-7\%) and malignancy (2-4\%) [1].

Depending on the cause of bleeding and anatomical localization, management of UGIB can be challenging. Standard methods for endoscopic hemostasis include mechanical, injection or thermal therapy. The current ESGE guideline on diagnosis and management of non-variceal UGIB recommends a com- 
bination of 2 modalities for management of peptic ulcer bleeding [1]. With a combination of these procedures, a hemostasis rate of $85 \%$ to $95 \%$ can be achieved, however, in about $5 \%$ to $10 \%$ of patients, rebleeding was reported [2].

TC-325 (Hemospray, Cook Medical) is a hemostatic powder approved for endoscopic hemostasis in the upper gastrointestinal tract. It consists of a mineral-based compound that after interacting with moisture (e.g. blood) becomes cohesive and adhesive, thus forming a tamponade covering the bleeding site. Through absorption of the fluid of the blood it leads to concentration of clotting factors and activates the clotting cascade [3]. Efficacy of TC-325 was initially shown in a pilot study in upper gastrointestinal peptic ulcer bleeding [4] and subsequently in various causes of gastrointestinal bleeding, ranging from cancer bleeding to acute variceal bleeding [5-9]. The SEALStudy was the first multicenter prospective registry demonstrating successful immediate hemostasis in $85 \%$ and a rebleeding rate of $15 \%$ in non-peptic-ulcer bleeding [10], thus confirming data from other smaller cohorts $[4,7]$. The overall primary hemostasis rate was otherwise reported to be in the range of $93 \%$ to $98 \%[2,6,7]$. In the current ESGE guideline for management of non-variceal UGIB, the role of TC-325 has not been clearly defined. Available data suggest that Hemospray is safe and effective and may be best used in high-risk cases as a temporizing measure or a bridge toward more definitive treatment [1]. Herein we report on our single-center experience with TC-325 in a 38-month period in patients with UGIB that was not amenable to standard endoscopic therapy or had persistent bleeding after standard endoscopic hemostasis.

\section{Patients and methods}

Since the introduction of TC-325 in November 2013, data has been collected on patients who have received TC-325 for endoscopic hemostasis at our center. Those data were analyzed retrospectively. This study was approved by the local institutional review board.

Indications for Hemospray use in our institution were: (1) UGIB that was not otherwise treatable (e.g. due to difficult anatomical situation or diffuse bleeding without definite sourcein those cases, Hemospray was used as monotherapy); and (2) application of Hemospray in UGIB as a salvage therapy after failure of other endoscopic methods.

Primary endpoints were: technical success (defined as successful immediate hemostasis after TC-325 application) and clinical success (defined as effective hemostasis and no recurrent bleeding in 7 days). Recurrent bleeding was diagnosed if evidence of fresh blood or signs of bleeding in the treated site was seen on follow-up endoscopy. Otherwise recurrent bleeding was defined if one of these criteria had been met: (1) hematemesis or melena; (2) a drop in hemoglobin $>2 \mathrm{mg} / \mathrm{dl}$ or transfusion of 4 or more blood packs; or (3) hemodynamic instability as previously described [4]. Secondary endpoints were recurrent bleeding until Days 3 and 7 , hospital mortality, and TC325-associated adverse events (AEs).

Complete Rockall-score was calculated for each patient to estimate the rebleeding risk as previously described [11].
Our center uses both standard and therapeutic endoscopes as part of standard protocol in acute bleeding situations. In patients with an expected major bleed, therapeutic endoscopes are used regularly. To avoid clogging of the working channel or kinking of the catheter, a $10 \mathrm{~F}$ catheter was applied through a therapeutic endoscope to the TC-325 cartridges used in this study. Due to the strict indications for the application of Hemospray in our unit, therapeutic endoscopes were generally already in use and no further endoscope change was needed. Second-look endoscopy was performed in patients with highrisk bleeding (e.g. Forrest la-Forrest Ilb ulcer bleeding) at the discretion of the endoscopist.

\section{Statistical analysis}

Patient data were collected prospectively in a database created with Microsoft Excel ${ }^{\mathrm{TM}}$. Statistical calculations were done with Microsoft Excel ${ }^{\mathrm{TM}}$ and with the Statistical Online Computational Resource (http://www.socr.ucla.edu/htmls). Descriptive statistics consisted of the mean, median, SD and range. Fisher's exact test was used to compare qualitative data. A $P$ value $<0.05$ was considered significant.

\section{Patient characteristics}

Fifty-two patients with a total of 67 TC-325 applications in the upper gastrointestinal tract were documented from November 2013 until February 2017. TC-325 was used in 23 of these patients $(44.2 \%)$ as a monotherapy in bleeding not amenable to standard endoscopic therapy and in 29 of the patients (55.8\%) as a salvage therapy after unsuccessful hemostasis with other endoscopic treatment modalities.

The median complete Rockall-score was 7 points (range 3 10). Twenty-seven of 52 patients (51.9\%) had a complete rockall-score of 7 or higher. Among the patients with a complete Rockall-score $<7$ points, 18 of 25 patients ( $72 \%$ ) had a score of 6 points.

The overall median age of the patients was 69 years (range 32-92 years) and most of the patients were male (31 males: 21 females). The most frequent comorbidity was malignancy (16/52 patients, $30.8 \%$ ), followed by atrial fibrillation and coronary heart disease (8/52 and 6/52 patients; $15.4 \%$ and $11.5 \%$, respectively). Thirteen patients ( $25 \%$ ) had salicylic acid as an antithrombotic therapy, whereas 13 patients (25\%) were on anticoagulation with either warfarin, heparin, rivaroxaban or apixaban. Details of the demographics, medications and relevant comorbidities are stated in $>$ Table 1.

The most frequent bleeding sources were peptic ulcers in 18 of 52 patients (34.6\%) (5 gastric ulcer and 13 duodenal ulcer), post-interventional ulcers (i.e. post EMR, ESD, EFTR) in 14/52 patients (27\%) and tumor bleeding in $10 / 52$ patients (19.2\%). The majority of the gastroduodenal ulcers were classified as Forrest Ib (7/18 patients; $38.9 \%$ ) and Forrest Ila (6/18 patients, $33.3 \%)$. One patient initially had a Forrest-Ila ulcer, which bled repeatedly despite standard endoscopic treatment. The ulcer was eventually stable and became a Forrest-III ulcer. In repeated EGD the ulcer "deteriorated" from a Forrest III to a Forrest IIc ulcer and it was deemed as a sign of rebleeding. Because con- 
- Table 1 Patient demographic and complete Rockall score, important comorbidities and medication in the whole cohort, monotherapy and salvage therapy subgroups. NSAID: non-steroidal anti-inflammatory drugs.

\begin{tabular}{|l|l|l|l|}
\hline & Total cohort (n= 52) & Monotherapy (n=23) & Salvage therapy (n=29) \\
\hline Age, $y$, median (range) & $69(32-92)$ & $68(32-92)$ & $69(36-89)$ \\
\hline Sex (m/f) & $31 / 21$ & $16 / 7$ & $15 / 14$ \\
\hline Platelet inhibition, $n$ (\%) & $13(25)$ & $9(39.1)$ & $4(13.8)$ \\
\hline Anticoagulation, $n$ (\%) & $13(25)$ & $3(13)$ & $10(34.5)$ \\
\hline NSAID, $n$ (\%) & $8(15.4)$ & $1(4.3)$ & $7(24.1)$ \\
\hline Glucocorticoid, $n$ (\%) & $1(1.9)$ & 0 & $1(3.4)$ \\
\hline Malignancy, $n$ (\%) & $16(30.8)$ & $4(17.4)$ & $12(41.4)$ \\
\hline Atrial fibrillation, $n$ (\%) & $8(15.4)$ & 0 & $8(27.6)$ \\
\hline Coronary heart disease, $n$ (\%) & $6(11.5)$ & $3(13)$ & $3(10.3)$ \\
\hline Complete Rockall score, median (range) & $7(3-10)$ & $7(5-9)$ & $7(3-10)$ \\
\hline$-<7, n(\%)$ & $25(48.1)$ & $11(47.8)$ & $14(48.3)$ \\
\hline \hline$\geq 7, n(\%)$ & $27(51.9)$ & & $15(51.7)$ \\
\hline
\end{tabular}

ventional endoscopic therapies had been exhausted, TC-325 was applied.

The mean diameter of the gastroduodenal ulcers was $28 \mathrm{~mm}$ (range $15-50 \mathrm{~mm}$ ). $>$ Table 2 summarizes the bleeding etiologies and location. Fig. 1 shows an example of TC-325's application in a duodenal ulcer.

The majority of the bleeding was located in the duodenum (29/52 patients, 55.8\%). Of those, 25 were located either in the bulb or in the second part of the duodenum (25/29 bleedings in duodenum $86.2 \%)$. Four bleeds (4/29, $13.8 \%$ ) were around the papilla vateri. In 16 of 52 patients (30.1\%), bleeding source was located in the stomach, 10 bleeds were located in the lesser curvature and 11 bleeds were located in the body of the stomach. There were 7 bleeds in the esophagus (13.5\%), all of which were in the distal third of the esophagus.

\section{Results}

Technical success (successful immediate hemostasis) was achieved in 51 of 52 patient (98.1\%). In 1 patient with postbanding variceal bleeding, Hemospray was used as a salvage therapy and failed to achieve hemostasis; the bleeding was stopped with an OTSC-application.

After 3 and 7 days, the overall rebleeding rate was $43.1 \%$ (22/51 patients) and 49\% (25/51 patients), respectively. The 3 -day rebleeding rate for patients who received TC-325 as salvage therapy was $44.8 \%$ (13/29 patients) whereas patients with monotherapy had a rebleeding rate of $39.1 \%$ (9/23 patients), $(P=0.78)$. After 7 days the rebleeding rates was $43.5 \%$ (10/23 patients) vs. $51.7 \%$ (15/29 patients) in the monotherapy and salvage therapy groups, respectively $(P=0.58)$.

The overall rebleeding rate on Day 3 for patients with peptic ulcer, post-interventional ulcer and tumor bleeding was $44.4 \%$ (8/18 patients), $50 \%$ (7/14 patients) and 20\% (2/10 patients), respectively. On Day 7 the rebleeding rate was $55.5 \%$ (10/18 patients), $57.1 \%$ (8/14 patients) and $20 \%$ (2/10 patients) for peptic ulcer, post-interventional ulcer and tumor bleeding, respectively.

Patients with peptic ulcer had a Day 3 rebleeding rate of $60 \%$ in the monotherapy group vs. $38.5 \%$ in the salvage therapy group ( $3 / 5$ patients vs. $5 / 13$ patients, $P=0.61$ ). The rebleeding rate for post-interventional ulcer on Day 3 was $25 \%$ in the monotherapy group vs. $60 \%$ in the salvage therapy (1/4 patients vs. $6 / 10$ patients, $P=0.56)$. The rebleeding rate for tumor bleeding on Day 3 was $28.6 \%$ vs. $0 \%$ (2/7 vs. $0 / 3$ patients, $P=1$ ). On day 7 there were 2 further bleeding cases from peptic ulcer in the salvage therapy group and 1 more bleed from a post-interventional ulcer in the monotherapy group. There were no changes in the tumor rebleeding rate on Day 7. Table 3 summarizes the overall rebleeding rate as well as rebleeding rate in the monotherapy and salvage therapy group after Days 3 and 7 for peptic ulcer, post-interventional ulcer and tumor bleeding. During further hospital stay, there was one patient with late rebleeding on Day 14 due to a mantle cell lymphoma.

Second-look endoscopy was performed in 42 of 52 patients (80.1\%) and was done mostly on the first post-interventional day (mean 1.3 days, range $1-12$ days). Rebleeding was seen in 19 patients (36.5\%). In 13 of 19 patients (68.4\%), Hemospray was applied once again. Acute hemostasis was again achieved in all these patients.

Mortality was $15.4 \%$ (8/52). Two deaths were bleedingrelated: 1 patient had terminal cancer with malignant bleeding and refused further chemotherapy. The other died of hemorrhagic shock. Other causes of mortality are stated in $>$ Table 4. There were no technical difficulties during all TC-325's application or other procedure-related AEs. 


\begin{tabular}{|c|c|}
\hline Bleeding etiology & n (\%) \\
\hline - Peptic ulcer & $18(34,6)$ \\
\hline - Post-interventional ulcer & $14(26,9)$ \\
\hline - Tumor bleeding & $10(19,2)$ \\
\hline - Endoscopic papillotomy & $2(3,8)$ \\
\hline - Reflux esophagitis & $2(3,8)$ \\
\hline - Post-variceal bleeding & $2(3,8)$ \\
\hline - GAVE-Syndrome & $1(1,9)$ \\
\hline - Dieulafoy’s lesion & $1(1,9)$ \\
\hline - Diffuse post-emesis bleeding & $1(1,9)$ \\
\hline - Jejunal and duodenal ischemia & $1(1,9)$ \\
\hline Bleeding & $n=52$ \\
\hline - Distal esophagus & 7 \\
\hline $\begin{array}{l}\text { - Gastric } \\
\text { - Cardia } \\
\text { - Body } \\
\text { - Antrum } \\
\text { - Lesser Curvature } \\
\text { - Greater Curvature }\end{array}$ & $\begin{array}{r}16 \\
1 \\
11 \\
4 \\
10 \\
5\end{array}$ \\
\hline $\begin{array}{l}\text { - Duodenal } \\
\text { - Duodenal bulb } \\
\text { - Papilla } \\
\text { - Descending part of the duodenum }\end{array}$ & $\begin{array}{r}29 \\
13 \\
4 \\
12\end{array}$ \\
\hline Localization of the peptic ulcer & $n=18$ \\
\hline - Gastric Ulcer & 5 \\
\hline - Duodenal Ulcer & 13 \\
\hline $\begin{array}{l}\text { Forrest classification for gastric and duodenal ulcer } \\
\text { bleeding }\end{array}$ & $n=18$ \\
\hline
\end{tabular}

- Forrest la (spurting)

- Forrest lb (oozing) 7

- Forrest lla (visible vessel) + evidence of bleeding 6

- Forrest IIb (adherent clot) 4

- Forrest Ilc (haematin on ulcer base)

Mean ulcer diameter $28 \mathrm{~mm}$ (Range $15-50 \mathrm{~mm}$ )

\section{Discussion}

We report our experience with TC-325 for treatment of upper gastrointestinal bleeding in 52 patients over a 38-month period. In our cohort, TC-325 was used as monotherapy for bleeding sources not amenable to standard methods or as "salvage" therapy after failure of endoscopic hemostasis. To our knowledge this study represents one of the largest single-center retrospective series. The study showed excellent applicability and technical success (98.1\%) but a high rate of rebleeding $(43.1 \%$ and $49 \%$ on Days 3 and 7 , respectively).
The high technical success rate in our study is in accordance with previous studies. Early studies and case series showed a constantly high technical success rate of $93 \%$ to $100 \%$ in various bleeding sources in the UGIB $[5-8,12,13]$ and LGIB [14]. The SEAL-Study was the first multicenter study on TC-325. It included 63 patients and showed a hemostasis rate of $85 \%$. The recently published French GRAPHE-Registry, which included 202 patients, revealed a high technical success rate of $>95 \%$, which confirmed results from early studies and case series [15]. Although our results confirm the effectiveness of TC-325 for immediate hemostasis, the high rebleeding rate after TC325 application is nevertheless concerning. Early reports have shown an overall rebleeding rate of $12 \%$ to $15 \%$ in mixed cohorts of UGIB and LGIB $[6,7,10]$. If only UGIB was considered, the rebleeding rate was higher. Chen et al. reported a rebleeding rate of $29.4 \%$ in patients with high-risk non-variceal UGIB within the first 72 hours and a total of $47 \%$ after $>72$ hours [6]. The GRAPHE-Registry showed a rebleeding rate of $26.7 \%$ on Day 8 and $33.5 \%$ on Day 30 [15]. Another study from Yau et al. also revealed a rebleeding rate of $39 \%$ within 7 days in 19 patients with UGIB [2], meanwhile Dixon et al. reported a $33 \%$ rate of rebleeding within 30 days in patients with UGIB [16]. Despite no directly comparable report, our results with a rebleeding rate of $49 \%$ within 7 days are grossly comparable to the literature.

One explanation for the high rebleeding rate in our study is the selection of patients. TC-325 was applied only in patients not amenable to conventional endoscopic therapy or as salvage therapy for patients after unsuccessful hemostasis with conventional methods (e.g. peptic ulcers with large diameter and/ or difficult anatomy). Fifty-two percent of our patients had a complete Rockall-score $>7$ and $86.5 \%$ had a score $>6$, indicating the high risk of re-bleeding in our cohort. The mean diameter of the gastroduodenal ulcers in our study was $28 \mathrm{~mm}$ (range $15-50 \mathrm{~mm}$ ) and the majority of the lesions were in the duodenum. In a meta-analysis [17] and the current ESGE guidelines [1], ulcer diameter $>20 \mathrm{~mm}$ and ulcer location in posterior duodenal wall are considered as predictors for endoscopic treatment failure $[1,17]$.

TC-325 becomes cohesive and adhesive after contact with blood or other moisture and mainly achieves hemostasis through formation of a mechanical barrier that adheres and covers the bleeding site. In the gastrointestinal tract with its constant peristaltic and mucus production, TC-325 is probably sloughed off the bleeding site within a few hours. When second-look endoscopy after 24 hours was performed, the powder was not visible in any patients in our study. The hemostatic effect of TC-325 thus is not as durable as other hemostasis methods such as the hemoclip.

Compared to other hemostasis topical powder such as EndoClot $^{\mathrm{TM}}$, TC-325 confers a higher primary hemostasis rate in UGIB $(64-100 \%[18,19]$ vs. $85-98 \%[2,6,7,10])$. The rebleeding rate with EndoClot ${ }^{\mathrm{TM}}$, however, was reported to be low at $4.8 \%$ to $11 \%$. Ankaferd BloodStopper ${ }^{\mathrm{TM}}$ (ABS), a topical hemostasis powder consisting a mixture of 5 different plant species, is available in Turkey and reportedly is effective for various casues of bleeding (e.g. diverticular bleeding [20], radiogenic esopha- 

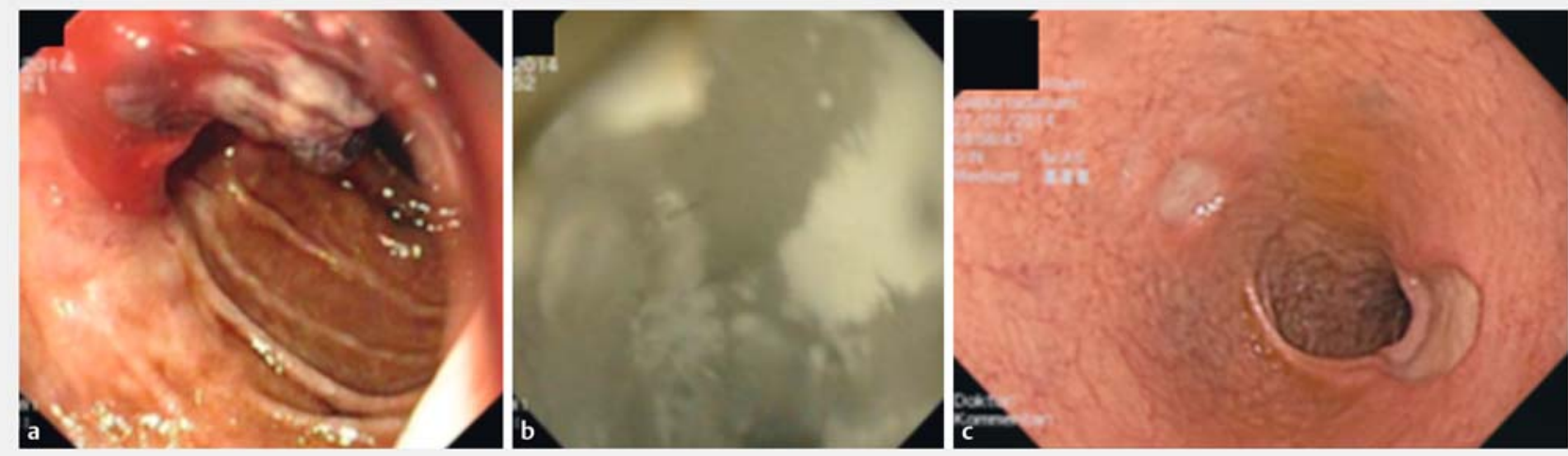

- Fig. 1 An example of TC-325 application in peptic ulcer. a The patient has a Forrest-lb bleeding from a duodenal ulcer with limited visibility, which was difficult to reach with mechanical hemostasis procedure or adrenalin injection. b Thus application of TC-325 was chosen as primary hemostasis method. c On second-look endoscopy, 2 duodenal ulcers with clean base (Forrest III) were visible without any further sign of bleeding.

Table 3 Overall rebleeding rate and rebleeding rate of the most frequent bleeding etiologies on day 3 and day 7.

\begin{tabular}{|c|c|c|c|c|}
\hline & Total cohort & Monotherapy & Salvage therapy & $P$ value \\
\hline Technical success, n (\%) & $51 / 52(98.1)$ & $23 / 23(100)$ & $28 / 29(96,6)$ & 1 \\
\hline Overall rebleeding on Day 3, n (\%) & $22 / 51(43.1)$ & $9 / 23(39.1)$ & $13 / 29(44.8)$ & 0.78 \\
\hline Overall rebleeding on Day 7, n (\%) & $25 / 51(49)$ & $10 / 23(43.5)$ & $15 / 29(51.7)$ & 0.58 \\
\hline Rebleeding from peptic ulcer on Day 3, n (\%) & $8 / 18(44.4)$ & $3 / 5(60)$ & $5 / 13(38.5)$ & 0.61 \\
\hline Rebleeding from peptic ulcer on Day 7, n (\%) & $10 / 18(55.5)$ & $3 / 5(60)$ & $7 / 13(54)$ & 1 \\
\hline Rebleeding from post-interventional ulcer on Day 3, n (\%) & $7 / 14(50)$ & $1 / 4(25)$ & $6 / 10(60)$ & 0.56 \\
\hline Rebleeding from post-interventional ulcer on Day $7, \mathrm{n}(\%)$ & $8 / 14(57.1)$ & $2 / 4(50)$ & $6 / 10(60)$ & 1 \\
\hline Rebleeding from tumor bleeding on Day 3, n (\%) & $2 / 10(20)$ & $2 / 7(28.6)$ & $0 / 3$ & 1 \\
\hline Rebleeding from tumor bleeding on Day $7, \mathrm{n}$ (\%) & $2 / 10(20)$ & $2 / 7(28.6)$ & $0 / 3$ & 1 \\
\hline
\end{tabular}

Table 4 Causes of mortality.

\begin{tabular}{|l|l|}
\hline Cause of mortality & $\mathbf{n}$ \\
\hline Bleeding-related* & 2 \\
\hline Sepsis & 1 \\
\hline Cerebral Ischemia & 1 \\
\hline Aspiration & 1 \\
\hline Not stated & 1 \\
\hline End-stage liver disease & 1 \\
\hline Ventricular fibrillation & 1 \\
\hline Total & 8 \\
\hline * See further description in text & \\
\hline
\end{tabular}

gitis [21] and dieulafoy's lesions [22]. Larger studies regarding ABS and EndoClot ${ }^{\mathrm{TM}}$ are lacking and needed before their widespread use.
In tumor bleeding the application of TC-325 as a hemostasis method is very promising due to its easy non-contact application and large coverage. Given diffuse bleeding and large bleeding area, conventional methods regularly have high bleeding rates ranging from $28 \%$ to $49 \%$ [23-25]. There were 10 tumor bleeds in this study, all of which had a $100 \%$ technical success rate and a clinical success rate of $80 \%$. This is comparable to outcomes previously reported $[6,15,26]$. A prospective study comparing TC-325 and other conventional methods in tumor bleeding is warranted to further validate these findings.

Based on the high rebleeding rates shown in our and other studies and the lack of randomized controlled trials comparing TC-325 with standard endoscopic methods, TC-325 may only be recommended as first-line endoscopic treatment for bleeding sources not amenable to conventional methods or as a salvage therapy. The role of Hemospray for first-line endoscopic treatment for bleeding sources accessible to standard methods as well as the question of whether it should primarily be used as monotherapy or combination therapy remains unclear. Current ESGE-guidelines recommend use of topical-spray (e. g. TC-325) only if bleeding was persistent or in recurrent bleeding [1]. Giv- 
en its easy application it may also be used in primary care units with limited experience in hemostasis as a bridge to definitive therapy in a next level care center.

Limitations of our study are the retrospective analysis and the heterogeneity of the patient cohort, as well as the lack of a control group. Furthermore, the results of this study can only be applied to the patient cohort described above and cannot be generalized for all patients with UGIB.

\section{Conclusion}

In conclusion, this study demonstrates easy application and high immediate efficacy of TC-325 as a monotherapy in UGIB not amenable to standard endoscopic therapy or as an add-on ("salvage") therapy after failure of other methods. However, rebleeding in this high-risk cohort was frequent. Randomized trials are warranted to compare TC-325 with standard endoscopic methods and to further define its role in the therapeutic algorithm.

\section{Competing interests}

None

References

[1] Gralnek IM, Dumonceau JM, Kuipers EJ et al. Diagnosis and management of nonvariceal upper gastrointestinal hemorrhage: European Society of Gastrointestinal Endoscopy (ESGE) Guideline. Endoscopy 2015; 47: a 1 - 46

[2] Yau AH, Ou G, Galorport C et al. Safety and efficacy of Hemospray(R) in upper gastrointestinal bleeding. Can J Gastroenterol Hepatol 2014; 28: $72-76$

[3] Barkun AN, Moosavi S, Martel M. Topical hemostatic agents: a systematic review with particular emphasis on endoscopic application in Gl bleeding. Gastrointest Endosc 2013; 77: 692 - 700

[4] Sung JJ, Luo D, Wu JC et al. Early clinical experience of the safety and effectiveness of Hemospray in achieving hemostasis in patients with acute peptic ulcer bleeding. Endoscopy 2011; 43: 291-295 doi:10.1055/s-0030-1256311

[5] Chen YI, Barkun AN, Soulellis C et al. Use of the endoscopically applied hemostatic powder TC-325 in cancer-related upper GI hemorrhage: preliminary experience (with video). Gastrointest Endosc 2012; 75: $1278-1281$

[6] Chen YI, Barkun A, Nolan S. Hemostatic powder TC-325 in the management of upper and lower gastrointestinal bleeding: a two-year experience at a single institution. Endoscopy 2015; 47: 167-171

[7] Sulz MC, Frei R, Meyenberger $C$ et al. Routine use of Hemospray for gastrointestinal bleeding: prospective two-center experience in Switzerland. Endoscopy 2014; 46: 619-624

[8] Ibrahim M, Lemmers A, Deviere J. Novel application of Hemospray to achieve hemostasis in post-variceal banding esophageal ulcers that are actively bleeding. Endoscopy 2014; 46: UCTN: E263
[9] Ibrahim M, El-Mikkawy A, Mostafa I et al. Endoscopic treatment of acute variceal hemorrhage by using hemostatic powder TC-325: a prospective pilot study. Gastrointest Endosc 2013; 78: 769- 773

[10] Smith LA, Stanley AJ, Bergman J] et al. Hemospray application in nonvariceal upper gastrointestinal bleeding: results of the Survey to Evaluate the Application of Hemospray in the Luminal Tract. J Clin Gastroenterol 2014; 48: e89-92

[11] Rockall TA, Logan RF, Devlin HB et al. Risk assessment after acute upper gastrointestinal haemorrhage. Gut 1996; 38: 316-321

[12] Smith LA, Morris A], Stanley AJ. The use of hemospray in portal hypertensive bleeding; a case series. J Hepatol 2014; 60: 457-460

[13] Parsi MA, Jang S. Hemospray for diffuse anastomotic bleeding. Gastrointest Endosc 2014; 80: 1170

[14] Holster IL, Brullet E, Kuipers EJ et al. Hemospray treatment is effective for lower gastrointestinal bleeding. Endoscopy 2014; 46: 75-78

[15] Haddara $S$, Jacques J, Lecleire $S$ et al. A novel hemostatic powder for upper gastrointestinal bleeding: a multicenter study (the "GRAPHE" registry). Endoscopy 2016; 48: 1084-1095

[16] Dixon S, Tate D, Przemioslo R et al. Hemospray may not reliably achieve hemostasis in acute upper gastrointestinal bleeding. Gut 2015; 64: A420.423 - A421

[17] Elmunzer B], Young SD, Inadomi JM et al. Systematic review of the predictors of recurrent hemorrhage after endoscopic hemostatic therapy for bleeding peptic ulcers. Am J Gastroenterol 2008; 103: 2625 - 2632; quiz 2633

[18] Prei JC, Barmeyer C, Burgel N et al. EndoClot Polysaccharide Hemostatic System in Nonvariceal Gastrointestinal Bleeding: Results of a Prospective Multicenter Observational Pilot Study. J Clin Gastroenterol 2016; 50: e95-e100

[19] Beg S, Al-Bakir I, Bhuva M et al. Early clinical experience of the safety and efficacy of EndoClot in the management of non-variceal upper gastrointestinal bleeding. Endosc Int Open 2015; 3: E605-609

[20] Aslan E, Akyuz U, Pata C. The use of Ankaferd in diverticular bleeding: two case reports. Turk J Gastroenterol 2013; 24: 441-443

[21] Ozturk O, Koklu S, Basar O et al. Severe radiation esophagitis successfully treated with Ankaferd hemostat. Gastrointest Endosc 2015; 81: $1048-1049$

[22] Kurt M, Onal IK, Akdogan M et al. Ankaferd Blood Stopper for controlling gastrointestinal bleeding due to distinct benign lesions refractory to conventional antihemorrhagic measures. Can J Gastroenterol Hepatol 2010; 24: $380-384$

[23] Kim YI, Choi IJ, Cho SJ et al. Outcome of endoscopic therapy for cancer bleeding in patients with unresectable gastric cancer. J Gastroenterol Hepatol 2013; 28: 1489-1495

[24] Lee J, Chung H. Clinical Outcomes of Endoscopic Hemostasis for Cancer Bleeding in Patients With Unresectable Advanced Gastric Cancer. Gastrointestinal Endoscopy81: AB246-AB247

[25] Sheibani S, Kim J], Chen B et al. Natural history of acute upper GI bleeding due to tumours: short-term success and long-term recurrence with or without endoscopic therapy. Aliment Pharmacol Ther 2013; 38: $144-150$

[26] Pittayanon R, Prueksapanich P, Rerknimitr R. The efficacy of Hemospray in patients with upper gastrointestinal bleeding from tumor. Endosc Int Open 2016; 4: E933-936 